\title{
Effect of Composition and Heat Treatment on MnBi
}

\section{Magnetic Materials}

\author{
Jun Cui ${ }^{\text {a }}$ Jung-Pyung Choi ${ }^{\text {a }}$ Evgueni Polikarpov ${ }^{\text {a }}$, Mark E Bowden ${ }^{\text {a }}$, Wei Xie ${ }^{\text {, }}$ \\ Guosheng Li ${ }^{\mathrm{a}}$, Zimin Nie ${ }^{\mathrm{a}}$, Nikolai Zarkevich ${ }^{\mathrm{b}}$, Mathew Kramer ${ }^{\mathrm{b}}$, Duane Johnson ${ }^{\mathrm{b}, \mathrm{c}}$ \\ ${ }^{a}$ Energy and Environment Directorate, Pacific Northwest National Laboratory/U.S. Department of \\ Energy, Richland, WA 99354, USA \\ ${ }^{\mathrm{b}}$ Division of Materials Science and Engineering, Ames Laboratory/U.S. Department of Energy, Ames, IA \\ 50011, USA \\ ${ }^{c}$ Department of Materials Science and Engineering, Iowa State University, Ames, IA 50011, USA
}

Corresponding author: Jun Cui, E-mail: jun.cui@pnnl.gov, TEL.: +1-509-375-2028.

\begin{abstract}
The metallic compound $\mathrm{MnBi}$ is a promising rare-earth-free permanent magnet material, unique among all candidates for its high intrinsic coercivity (Hci) and its large positive temperature coefficient. The Hci of MnBi in thin-film or in powder form can exceed $12 \mathrm{kOe}$ and $26 \mathrm{kOe}$ at $300 \mathrm{~K}$ and $523 \mathrm{~K}$, respectively. Such a steep rise in Hci with increasing temperature is unique to MnBi. Consequently, MnBi is a highly sought-after hard phase for exchange coupling nanocomposite magnets. However, the reaction between $\mathrm{Mn}$ and $\mathrm{Bi}$ is peritectic, so $\mathrm{Mn}$ tends to precipitate out of the MnBi liquid during the solidification process. As result, when the alloy is prepared using conventional induction or arc-melting casting methods, additional $\mathrm{Mn}$ is required to compensate the precipitation of $\mathrm{Mn}$. In addition to composition, post-casting annealing plays
\end{abstract}

C) 2014. This manuscript version is made available under the Elsevier user license http://www.elsevier.com/open-access/userlicense/1.0/ 
an important role in obtaining high content of $\mathrm{MnBi}$ low-temperature phase (LTP) because the annealing encourages the Mn precipitates and the unreacted Bi to react forming the desired LTP phase. Here we report a systematic study of the effect of composition and heat treatments on the phase content and magnetic properties of Mn-Bi alloys. In this study, 14 compositions were prepared using conventional metallurgical methods, and the resulting alloys were analyzed for compositions, crystal structures, phase content, and magnetic properties. The results show that the composition with 55 at.\% Mn exhibits both the highest LTP content (93 wt.\%) and magnetization (74 emu/g with 9T applied field at $300 \mathrm{~K})$.

Keywords: MnBi, rare-earth free, permanent magnet

\section{Introduction}

MnBi compound is a promising permanent magnetic material because of its high coercivity with a large positive temperature coefficient. MnBi exhibits a first-order structural transition between temperatures of $613 \mathrm{~K}\left(340{ }^{\circ} \mathrm{C}\right)$ and $628 \mathrm{~K}\left(355^{\circ} \mathrm{C}\right)$ [1]. The equilibrium phase below the transition point is commonly referred to as the low-temperature phase (LTP) and that above is the high-temperature phase (HTP). The composition, lattice structure, and parameters for the HTP phase are $\mathrm{Mn}_{2.23} \mathrm{Bi}_{1.88}, \mathrm{Pmma}$, and $\mathrm{a}=0.5959 \mathrm{~nm}, \mathrm{~b}=0.4334 \mathrm{~nm}, \mathrm{c}=0.7505 \mathrm{~nm}, \alpha=\beta=\gamma$ $=90^{\circ}$, respectively [2]. For the LTP phase, they are $\mathrm{Mn}_{50} \mathrm{Bi}_{50}, \mathrm{P} 63 / m m c$, and $\mathrm{a}=\mathrm{b}=0.4290 \mathrm{~nm}$, $\mathrm{c}=0.6126 \mathrm{~nm}, \alpha=\beta=90^{\circ}, \gamma=120^{\circ}$, respectively [3-5]. The LTP MnBi has a high magnetocrystalline anisotropy of $\left(1.6 \times 10^{6} \mathrm{~J} / \mathrm{m}^{3}\right)$, and relatively low saturation magnetization $(81 \mathrm{emu} / \mathrm{g}, \sim 9 \mathrm{kG})$ at $300 \mathrm{~K}[6]$. Its theoretical maximum energy product is estimated to be 20 MGOe using the formula $(\mathrm{BH})_{\max }=\mathrm{M}_{\mathrm{s}}{ }^{2} / 4$, where $\mathrm{M}_{\mathrm{s}}$ is the saturation magnetization. The first 
bulk MnBi magnet was prepared by Adams in 1953 [7]; an energy product of 5.3 MGOe (42 $\mathrm{kJ} / \mathrm{m}^{3}$ ) was achieved.

Part of the reason for such a low energy product compared to its theoretical maximum value was attributed to the difficulty of obtaining a high volume fraction of the desired LTP MnBi. The difficulty originates from the peritectic reaction between $\mathrm{Mn}$ and $\mathrm{Bi}$, which causes $\mathrm{Mn}$ to solidify and segregate from the Mn-Bi liquid during the solidification process. The kinetics of the segregated Mn reacting with the excessive Bi to form LTP MnBi is slow [8]. Researchers tried to make MnBi alloy with high LTP content using various metallurgical methods, but all failed to exceed 90 wt.\% [8-12].

In 1990, melt-spinning method was adopted for MnBi synthesis, and over 95 wt.\% LTP MnBi was achieved [13-17]. This great achievement is attributed to the high cooling rate of the meltspinning method, which is generally believed to be in excess of $10^{6} \mathrm{~K} / \mathrm{s}$. While it is common for a small (10-gram) capacity melt-spinning setup to reach the cooling rate necessary to obtain a MnBi ribbon free of Mn precipitates, it is rather difficult for a larger (kilogram or higher) capacity setup to achieve the same. A conventional metallurgical method, such as arc or induction melting casting followed by thermal-mechanical treatment, is much preferred for feasibility and cost effectiveness. We have been developing such a method for over two years, and reported the properties of the resulting high-purity powder with composition near 56 at.\% Mn $[18,19]$. The same method was used to prepare a series of Mn-Bi alloys to optimize composition for maximum magnetization. This paper presents details of this conventional metallurgical method and the result of composition optimization. 


\section{Experimental}

\subsection{Sample preparation}

High purity Mn (manganese) chips (Alfa Aesar: 99.98 wt.\%) and Bi (bismuth) shot (Aldrich: 99.999 wt.\%) were weighed according to the designed atomic ratio, then mixed and pressed to form a pellet using a die with $19 \mathrm{~mm}$ inner diameter. The designed weight of each pellet is about $20 \mathrm{~g}$; the actual weights of the as-obtained pellets vary, but are within $\pm 0.1 \mathrm{~g}$ error. In total, 14 pellets were prepared. The designed composition varied from 45 to 80 at. $\% \mathrm{Mn}$. These pellets were arc melted and cast into buttons.

\subsection{Heat treatments}

The LTP MnBi is sensitive to oxygen. According to the FactSage thermochemical database, the change in free energy for the $\mathrm{MnBi}$ oxidization reaction, $3 \mathrm{MnBi}+4.25 \mathrm{O}_{2}(\mathrm{~g})=\mathrm{Mn}_{3} \mathrm{O}_{4}+$ $1.5 \mathrm{Bi}_{2} \mathrm{O}_{3}$ is $-156 \mathrm{kcal} / \mathrm{mol}$ at $300 \mathrm{~K}\left(27^{\circ} \mathrm{C}\right)$. It appears that $\mathrm{MnBi}$ alloy is more oxygen sensitive than $\mathrm{Cu}(-130 \mathrm{kcal} / \mathrm{mol})$. Hence, heat treatment of MnBi alloys must be carried out in an inert or reducing atmosphere.

The as-obtained buttons were heat-treated using a tube furnace under pure hydrogen gas atmosphere. The heat-treatment schedule had two steps: 1$)$ ramp to $528 \mathrm{~K}\left(255^{\circ} \mathrm{C}\right)$ at a rate of 10 $\mathrm{K} / \mathrm{min}$, hold for 8 hours; and 2) ramp to $625 \mathrm{~K}\left(352^{\circ} \mathrm{C}\right)$ at a rate of $10 \mathrm{~K} / \mathrm{min}$, hold for 5 hours, then furnace cool to room temperature. The second step dwells at a temperature between the LTP and HTP MnBi formation temperatures of $613-628 \mathrm{~K}\left(340-355^{\circ} \mathrm{C}\right)$. During this step, some LTP decomposes to liquid and HTP. However, the decomposition is expected to be small, and higher temperature is advantageous for the $\mathrm{Mn}$ and $\mathrm{Bi}$ precipitates to react. 
Following the heat treatment, the buttons were crushed, ground and sieved to powders with three sizes ( $>44 \mu \mathrm{m}, 37-44 \mu \mathrm{m}$, and $<37 \mu \mathrm{m}$ ). The $37-44 \mu \mathrm{m}$ powder was further heat treated under vacuum ( $100 \mathrm{mTorr})$ at $563 \mathrm{~K}\left(290{ }^{\circ} \mathrm{C}\right)$ for 24 hours.

\subsection{Characterization}

Compositions of the as-obtained samples were analyzed using inductively coupled plasma atomic emission spectroscopy (ICP-AES). X-ray diffraction patterns of the powders were collected with a Panalytical X-Pert Bragg-Brentano diffractometer. A $0.05 \mathrm{~mm}$ receiving slit was used for increased resolution. Phase proportions and cell parameters were determined by wholepattern Rietveld fitting using the fundamental-parameters approach implemented in Topas (v4.2, Bruker AXS) and crystal structures from the Inorganic Crystal Structure Database. Magnetic properties of the powders were characterized using vibrating sample magnetometer (VSM) and physical properties measurement system (PPMS). Microstructures of the ingots and morphology of the powders were analyzed using scanning electron microscope (SEM).

\section{Experimental results}

\subsection{Microstructures of the MnBi ingots before and after the two-step heat treatment}

Microstructures of the as-cast ingots with 45, 55, and 75 at.\% Mn are shown in Fig. 1. The microstructures of these as-cast ingots show areas with three distinct colors: black, dark gray and light gray. Composition analysis shows that these colors correspond to $\mathrm{Mn}, \mathrm{MnBi}$, and Bi phases, respectively. Fig 1(a) shows that despite the ingot being 5 at.\% Mn less than the stoichiometric point, noticeable amount of $\mathrm{Mn}$ phase can be still be found in the ingot. It is apparent that the cooling rate of the arc-melting casting process is not fast enough to prevent Mn from 
precipitating. According to the Mn-Bi phase diagram shown in Fig. 2, the MnBi alloy with 45 at.\% Mn remains liquid when the temperature is above $\sim 1133 \mathrm{~K}\left(860{ }^{\circ} \mathrm{C}\right)$. If the liquid is quenched to below $535 \mathrm{~K}\left(262{ }^{\circ} \mathrm{C}\right)$, the ingot will have a small amount of $\mathrm{Bi}$ and a large amount of $\mathrm{MnBi}(\sim 90$ at.\%). It will be free of Mn precipitate. In contrast, if the liquid is gradually cooled to below $535 \mathrm{~K}\left(262{ }^{\circ} \mathrm{C}\right)$, as much as 32 at.\% of Mn may precipitate out and coexist with LTP and $\mathrm{Bi}$ phases in the ingot at room temperature. The phase diagram suggests the amount of $\mathrm{Mn}$ precipitates increases with increasing Mn content in the starting Mn-Bi mixture. This trend is evident by the increasing size of the Mn areas in the ingots with 55 and 75 at.\% Mn, as shown in Fig. 1(b) and 1(c), respectively.

Microstructures of the heat-treated ingots are shown in Fig. 3. A significant increase of LTP MnBi phase (gray area) is observed for all three compositions. For the ingot with 45 at.\% Mn, Fig. 3(a) shows that Mn metal still exists after the heat treatment, and the Mn metal is embedded in the LTP, suggesting it is the product of HTP to LTP transformation. For the ingot with 55 at.\% Mn, Fig. 3(b) shows that the LTP is uniformly distributed and forms a matrix. The Bi phase is successfully minimized, but the amount of Mn metal appears to be significant and the formation of these Mn metal areas appears to be dendritic and embedded in the LTP. For the ingot with 75 at.\% Mn, Fig. 3(c) shows significantly larger Mn areas. Interestingly, Bi areas are also observed despite the composition being far off the stoichiometric point. The hypothesis for Bi deposition is that forming large areas of $\mathrm{Mn}$ phase may cause nearby solution to be rich in $\mathrm{Bi}$, which subsequently forms Bi phase. Fig. 3(c) shows most of the Bi areas (light gray in color) are near the Mn region (black in color).

\subsection{Effect of grind-and-sieve and vacuum heat treatment on LTP content}


After the two-step heat treatment, the ingots were ground into powders (37 44 $\mu \mathrm{m})$, then heat treated under vacuum $(\sim 100 \mathrm{mTorr})$ at $563 \mathrm{~K}\left(290{ }^{\circ} \mathrm{C}\right)$ for 24 hours to encourage the precipitated Mn and Bi to form more LTP MnBi. Fig. 4 shows the morphology of the powder before and after vacuum heat treatment. Similarity in size and shape of the particles before and after vacuum heat treatment implies little sintering occurred during the vacuum heat treatment.

To examine the effect of the vacuum heat-treatment on compositions and phase fractions of MnBi alloy, ICP-AES and XRD analysis were carried out for the powders. Fig. 5 shows the amount of excessive Mn before and after the vacuum heat treatment measured by ICP-AES. The compositions of the arc melted buttons and the heat-treated powders systematically deviate from the designed composition (i.e., the composition of the pellets before arc melting). Below the composition of 55 at. $\% \mathrm{Mn}$, the alloys tend to have more Mn than they are formulated to. For example, the pellet with 45 at.\% Mn gained 2.2 at.\% Mn (lost 2.2 at.\% Bi) after the arc melting. Above the composition of 55 at.\% Mn, the alloys tend to have less Mn than they are formulated to. For example, the pellet with 80 at.\% Mn lost 3.4 at.\% Mn after arc melting.

There are 4 steps to process a MnBi ingot to the powder containing high content of LTP MnBi. These steps are arc melting, two-step heat treatment in hydrogen, grind-\&-seive, and vacuum heat-treatment. Figure 6 shows the XRD patterns of the powders after each processing step for the alloy with 55 at.\% Mn. It shows that the intensity of the Bi peaks gradually decreases and eventually disappears as processing progresses. The phase fractions of each composition, before and after heat treatment, are summarized in Table 1 and plotted in Fig. 7. The plot in Fig. 7(a) shows that after the grind-and-sieve process, Bi decreases with increasing Mn content, almost linearly but with small fluctuation in the beginning (Mn $<55$ at.\%); Mn increases more linearly with increasing Mn content. More importantly, the amount of MnBi phase fluctuates around 65 
mol.\% before the Mn content exceeds 55 at.\%, then linearly decreases with increasing Mn content. The overall trend of MnBi content is decreasing. Fig. 7(b) shows the phase fractions after the vacuum heat treatment. Clearly, the monotonic trends are broken and an optimum composition point emerges. First of all, most of the Mn phase is converted to $\mathrm{MnO}$, and the percentage of $\mathrm{MnO}$ is relatively constant and independent of $\mathrm{Mn}$ content, indicating the kinetics of the Mn oxidization process is related to the heat treatment process. Second, Bi quickly decreases to less than 2 mol.\% at 55 at.\% Mn, then remains constant. More importantly, the amount of MnBi quickly increases to 80 mol.\% at 55 at.\% Mn, then decreases linearly with increasing Mn content.

\subsection{Magnetic properties of the MnBi powders}

The magnetizations of the MnBi powders versus compositions at $300 \mathrm{~K}\left(27^{\circ} \mathrm{C}\right)$ are plotted in

Fig. 8. The applied field was $2.3 \mathrm{~T}$, which far exceeded the typical coercivity of these powders $(<$ $3 \mathrm{kOe}$ ). Our experience for $\mathrm{MnBi}$ indicates that the particle size has to be less than $5 \mu \mathrm{m}$ to show coercivity greater than $10 \mathrm{kOe}$. If the MnBi particle size is big (e.g., 25-40 $\mu \mathrm{m}$ ), the highest coercivity it exhibits is about $3 \mathrm{kOe}$. Not surprisingly, Figure 8 shows the composition with 55 at.\% Mn shows the highest magnetic moment given the fact that this is the composition possessing the highest LTP MnBi content, while the compositions away from this value show significantly reduced magnetic moments. Figure 9 shows the MH loop of the MnBi powder with 55 at.\% Mn composition with 9 T field. It shows the saturation magnetization of the 55 at.\% Mn powder is about $74 \mathrm{emu} / \mathrm{g}$ at $300 \mathrm{~K}$. Recall that the LTP MnBi content in this powder is about 93 wt.\%; a powder with $100 \%$ LTP MnBi would show a saturation magnetization of $80 \mathrm{emu} / \mathrm{g}$. 


\section{Discussions}

\subsection{Deviation of the obtained compositions from the designed value}

We think that the deviation of the as-obtained composition from the designed value is related to the grind-and-sieve process that removes Bi metal from the sample. The grinding process fractures particles to expose $\mathrm{Bi}$, and then presses the exposed soft $\mathrm{Bi}$ onto the mortar wall, forming a dark metallic colored coating. The more Bi metal in the crushed MnBi powder the more $\mathrm{Bi}$ metal is removed by the grind-and-sieve process. In addition to Bi removal, the grinding process also removes $\mathrm{Mn}$ metal by first forming $\mathrm{MnO}$, which is hard and easily broken into fine powder, then pressed the $\mathrm{MnO}$ powder into the soft Bi metal coating on the mortar. The residual on the mortar wall was analyzed for its composition using ICP-AES. It shows both Mn and Bi elements are present in the residual with $\mathrm{Mn}: \mathrm{Bi}$ mole ratio close to 0.5 . It appears that the grindand-sieve process removes both $\mathrm{Bi}$ and $\mathrm{Mn}$. It is a competing process during which Bi removal dominates below the 55 at.\% Mn point while above that point Mn removal starts to take over.

\subsection{Importance of HTP to LTP phase transformation}

The HTP has about 54.3\% at.\% Mn. It transforms to LTP and Mn between $613 \mathrm{~K}\left(340{ }^{\circ} \mathrm{C}\right)$ and $628 \mathrm{~K}\left(355^{\circ} \mathrm{C}\right)$. The 55 at. $\%$ Mn optimum point is thought to be closely related to this transformation. For the alloys with compositions $<55$ at.\% Mn during the solidification process, the alloy first forms the Mn phase, the HTP and a Bi-rich liquid phase. Then, the HTP transforms to the LTP and Mn. In the mean time, the Bi-rich liquid transforms to the LTP and a liquid phase containing even more $\mathrm{Bi}$, of which eventually decomposes to the $\mathrm{Bi}$ and the LTP. For the alloys with compositions $>55$ at. $\% \mathrm{Mn}$, the phase formation process is similar. The difference lies in 
the phase ratios. Because the starting composition is on the right side of the HTP, the amount of the Bi-rich liquid is small and the resulting Bi phase is small. Apparently, the HTP is an intermediate phase that cannot be avoided during the cast-chill solidification process. Fundamentally, the reason for preferential formation of HTP is that the energy barrier for Mn to diffuses in the quenched HTP (a partially-disordered structure with fractional occupancies) is lower than that in the LTP (a fully-ordered structure). Therefore, to maximize the LTP content, excessive $4.3 \%$ of Mn must be added to maximize the HTP content. A possible way to bypass formation of the HTP is to precipitate the LTP directly from the Bi-rich melt with $<10$ at.\% Mn. In addition, the calculated energy barriers in the LTP for diffusion of Mn and Bi vacancies and interstitials at $0 \mathrm{~K}$ are all above $1 \mathrm{eV}$. Although these barriers become lower with increasing temperature, they are too high compared to $k \mathrm{~T}<53 \mathrm{meV}$ at $613 \mathrm{~K}\left(340{ }^{\circ} \mathrm{C}\right)$. Consequently, diffusion of Mn and Bi to the LTP is not expected, explaining the sluggish kinetic of the reaction $\mathrm{Mn}+\mathrm{Bi}=\mathrm{MnBi}$.

\subsection{Weight changes of the phases during vacuum heat treatment}

To understand the weight changes of these phases during the vacuum heat treatment, one can use the XRD phase fraction analysis in Table 1 and the mole ratios of the two reactions to derive approximate weight changes. For example, for the sample with 55 at.\% Mn (designed composition), one gram of powder consists of $0.82 \mathrm{~g}$ of $\mathrm{MnBi}$ phase, $0.14 \mathrm{~g}$ of Bi phase, $0.05 \mathrm{~g}$ of $\mathrm{Mn}$ phase, and $0 \mathrm{~g}$ of $\mathrm{MnO}$. Assume that during the vacuum heat treatment, the following occur: 1) Y g of Bi is vaporized and taken away by vacuum; 2) Z g of Bi reacts with $\mathrm{Q} g$ of $\mathrm{Mn}$ phase, forming X g of $\mathrm{MnBi}$; 3) $\mathrm{P}$ g of Mn reacts with oxygen, forming $\mathrm{N}$ g of $\mathrm{MnO}$ phase. In addition, during each vacuum heat treatment run, the powder is weighed and the values before 
and after the treatment are compared. It was found that for the sample with 55 at.\% $\mathrm{Mn}$ (designed composition), the total weight change of the powder is negligible $(<0.05 \mathrm{wt} . \%)$, therefore the sum of the weight changes for these phases can be set to zero, i.e.,

$$
X-Y-Z-P-Q+N=0
$$

Moreover, the loss of $\mathrm{Bi}$ to vacuum, Y, must be equal to the gain of oxygen; therefore,

$$
\mathrm{N} \times \mathrm{MW}_{\mathrm{O}} / \mathrm{MW}_{\mathrm{MnO}}=Y,
$$

where $\mathrm{MW}_{\mathrm{MnO}}$ and $\mathrm{MW}_{\mathrm{O}}$ are the molecular weight of $\mathrm{MnO}$ and $\mathrm{O}$, respectively. Furthermore, when the $\mathrm{Bi}$ and $\mathrm{Mn}$ react to form $\mathrm{MnBi}$, their mole numbers must be equal; therefore,

$$
Z / \mathrm{MW}_{\mathrm{Bi}}=Q / \mathrm{MW}_{\mathrm{Mn}}=X / \mathrm{MW}_{\mathrm{MnBi}},
$$

where $\mathrm{MW}_{\mathrm{Bi}}, \mathrm{MW}_{\mathrm{Mn}}$, and $\mathrm{MW}_{\mathrm{MnBi}}$ are the molecular weights of the $\mathrm{Bi}, \mathrm{Mn}$ and $\mathrm{MnBi}$ phases, respectively. Similarly, the mole number of the $\mathrm{Mn}$ phase reacting with oxygen to form $\mathrm{MnO}$ is equal to the mole number of the $\mathrm{MnO}$ phase:

$$
P / \mathrm{MW}_{\mathrm{Mn}}=N / \mathrm{MW}_{\mathrm{MnO}} \text {. }
$$

Lastly, according to Table 1, the weights of the $\mathrm{MnBi}$ and $\mathrm{MnO}$ phases before and after vacuum heat treatment are

$$
\begin{aligned}
& 0.82+X=0.93, \\
& N=0.04
\end{aligned}
$$

Based on the above equations, the six variables can be solved. The solution is $\mathrm{X}=0.11 \mathrm{~g}, \mathrm{Y}=$ $0.01 \mathrm{~g}, \mathrm{Z}=0.09 \mathrm{~g}, \mathrm{P}=0.03 \mathrm{~g}, \mathrm{Q}=0.02 \mathrm{~g}, \mathrm{~N}=0.04 \mathrm{~g}$. The interpretation of this solution is that during the vacuum heat treatment of $1 \mathrm{~g}$ of 55 at.\% Mn powder, $0.09 \mathrm{~g}$ of Bi and $0.02 \mathrm{~g}$ of $\mathrm{Mn}$ reacted together, forming $0.11 \mathrm{~g}$ of $\mathrm{MnBi} ; 0.01 \mathrm{~g}$ of $\mathrm{Bi}$ is lost to vacuum; and $0.03 \mathrm{~g}$ of $\mathrm{Mn}$ reacted with $0.01 \mathrm{~g}$ of oxygen, forming $0.04 \mathrm{~g}$ of $\mathrm{MnO}$. The lost $\mathrm{Bi}$ has the same weight as the gained oxygen, resulting in zero overall weight change. The calculation further indicates that the 
weight of the remaining Bi metal is about $0.04 \mathrm{~g}$ after heat treatment; this is reasonably close to the $0.02 \mathrm{~g}$ value observed by XRD, which has an error range of $\pm 0.01 \mathrm{~g}$.

The main conclusion of this calculation is that the gain in LTP-MnBi phase fraction during the vacuum heat treatment is through the reaction of $\mathrm{Mn}$ and $\mathrm{Bi}$. Vacuum helps but is not critical. If oxygen can be tightly controlled, it is possible to use unreacted Mn and Bi more effectively, and the optimum composition will be closer to the stoichiometric point. For example, with 52 at.\% Mn composition, there is $4 \mathrm{wt} . \% \mathrm{MnO}$ and $6 \mathrm{wt} . \% \mathrm{Bi}$ in the heat-treated sample. These can form $\mathrm{MnBi}$ if oxygen is not present.

\section{Conclusions}

MnBi with LTP content is difficult to obtain because of the peritectic reaction between Mn and $\mathrm{Bi}$, where $\mathrm{Mn}$ phase starts to separate during the solidification process. Due to the preferential formation of the HTP, which later transforms to LTP and Mn, excess Mn is required to compensate the Mn precipitation. A newly developed thermo-mechanical processing route is shown to be able to synthesize MnBi powder with > 90 wt.\% LTP. The process takes advantage of the differences in physical properties of the phases coexisting in the cast MnBi alloys, such as hardness and vapor pressure, to remove the unreacted phases. The process also takes into account the excessive Mn required to compensate the Mn precipitation, therefore maximizing the amount of LTP MnBi. The process is easy to implement and compatible with the current industrial practice. In addition, for the developed process, 55 at.\% Mn was identified as the optimum composition for the MnBi alloy with the highest LTP content and the highest magnetization. 


\section{Acknowledgements}

This research was supported by the U.S. Department of Energy (DOE) Advanced Research

Projects Agency-Energy (ARPA-E) under Contract No. 11/CJ000/09/03. Ames Laboratory is

operated for the U.S. DOE by Iowa State University under contract \#DE-AC02-07CH11358.

\section{References}

[1] Chen T, Stutius WE. Ieee T Magn 1974;Ma10:581.

[2] Cenzual K, Gelato LM, Penzo M, Parthe E. Acta Crystallogr B 1991;47:433.

[3] Cui J, Choi JP, Li G, Polikarpov E, Darsell J, Kramer MJ, Zarkevich NA, Wang LL, Johnson DD, Marinescu M, Huang QZ, Wu H, Vuong NV, Liu JP. Journal of Applied Physics 2014;115.

[4] Andresen AF, Halg W, Fischer P, Stoll E. Acta Chem Scand 1967;21:1543.

[5] Yang JB, Yelon WB, James WJ, Cai Q, Roy S, Ali N. Journal of Applied Physics 2002;91:7866.

[6] Guillaud C. J Phys-Paris 1951;12:143.

[7] Adams E. Rev Mod Phys 1953;25:306.

[8] Andresen AF. Acta Chemica Scandinavica 1967;21:1543.

[9] van Goor JMN. Journal of Applied Physics 1968;39:5471.

[10] Pirich RG. Ieee T Magn 1980;16:1065.

[11] Rao NVR, Gabay AM, Hadjipanayis GC. Journal of Physics D-Applied Physics 2013;46.

[12] Rao NVR, Gabay AM, Li WF, Hadjipanayis GC. Journal of Physics D-Applied Physics 2013;46.

[13] Guo X, Zaluska A, Altounian Z, Stromolsen JO. J Mater Res 1990;5:2646.

[14] Guo X, Zaluska A, Altounian Z, Stromolsen JO. Mat Sci Eng a-Struct 1991;133:509.

[15] Guo X, Chen X, Altounian Z, Stromolsen JO. Phys Rev B 1992;46:14578.

[16] Yang JB, Yelon WB, James WJ, Cai Q, Kornecki M, Roy S, Ali N, l'Heritier P. J Phys-Condens Mat 2002;14:6509.

[17] Yang JB, Yelon WB, James WJ, Cai Q, Roy S, Ali N. J Appl Phys 2002;91:7866.

[18] Cui J, Choi JP, Li G, Polikarpov E, Darsell J, Kramer MJ, Zarkevich NA, Wang LL, Johnson DD, Marinescu M, Huang QZ, Wu H, Vuong NV, Liu JP. Journal of Applied Physics 2014;115:17A743.

[19] Cui J, Choi JP, Li G, Polikarpov E, Darsell J, Overman N, Olszta M, Schreiber D, Bowden M, Droubay T, Kramer MJ, Zarkevich NA, Wang LL, Johnson DD, Marinescu M, Takeuchi I, Huang QZ, Wu H, Reeve H, Vuong NV, Liu JP. Journal of physics. Condensed matter : an Institute of Physics journal 2014;26:064212. 

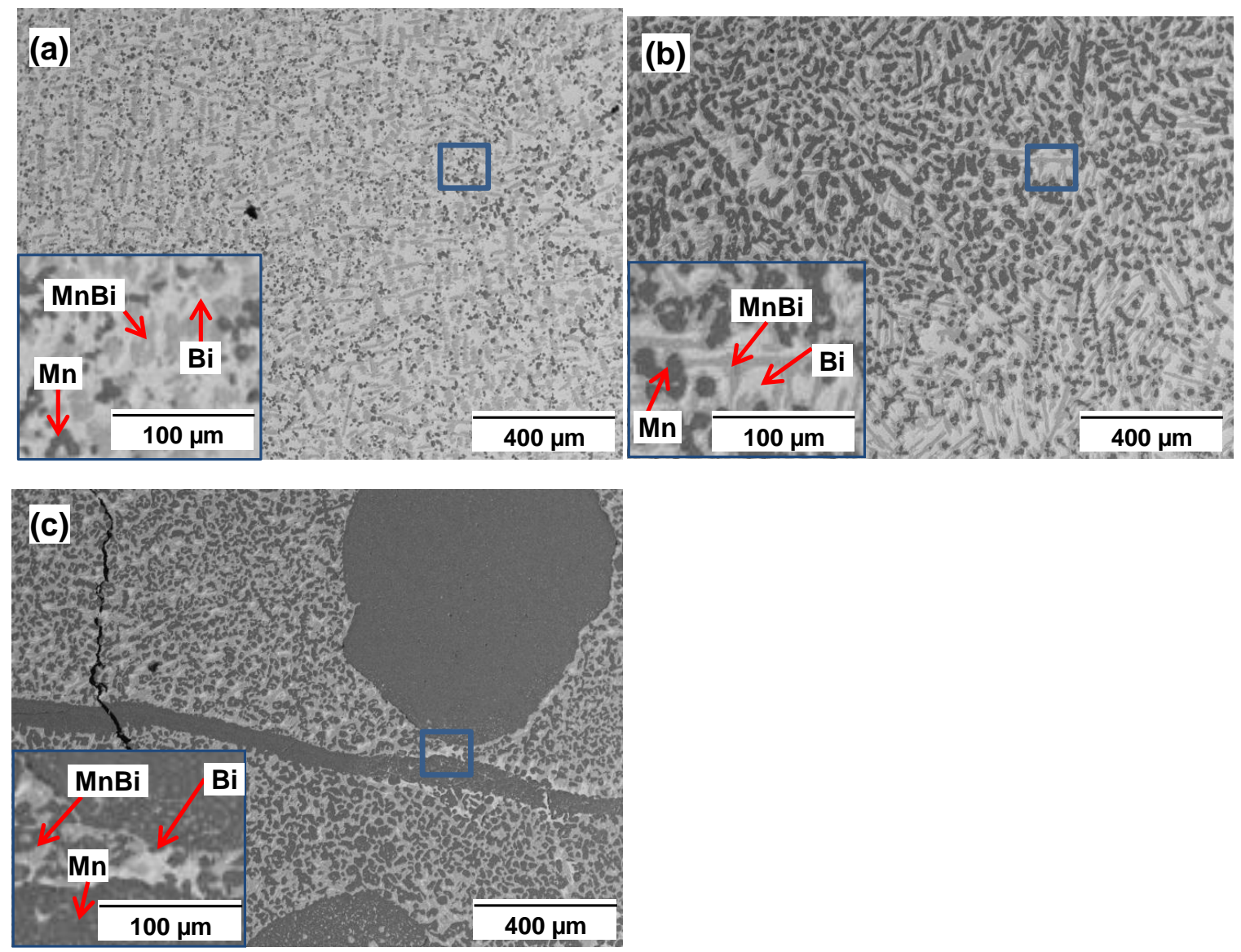

Figure 1. The microstructure of as-cast MnBi ingots obtained by arc melting.

(a) 45 at. $\% \mathrm{Mn}$, (b) 55 at. $\% \mathrm{Mn}$, and (c) 75 at.\% Mn 


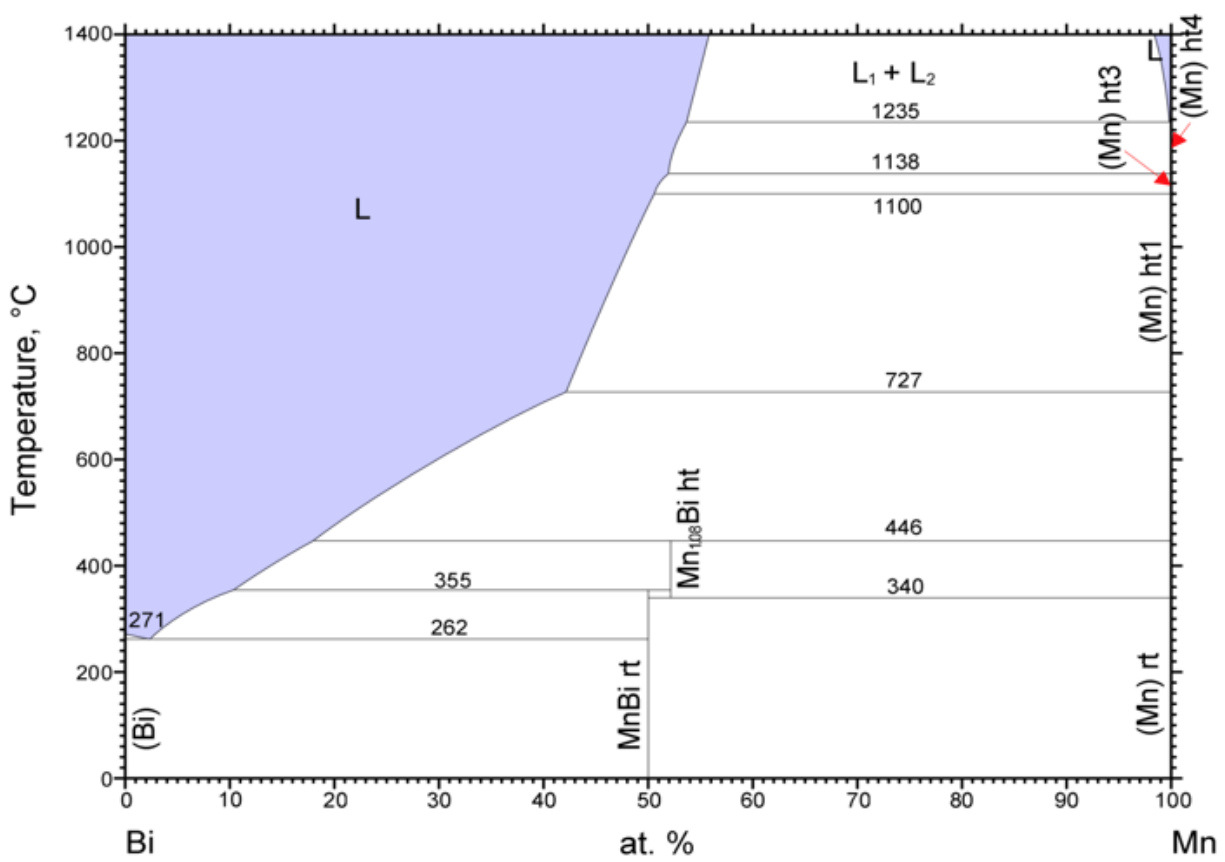

Figure 2. The phase diagram of Bi-Mn [1]. 

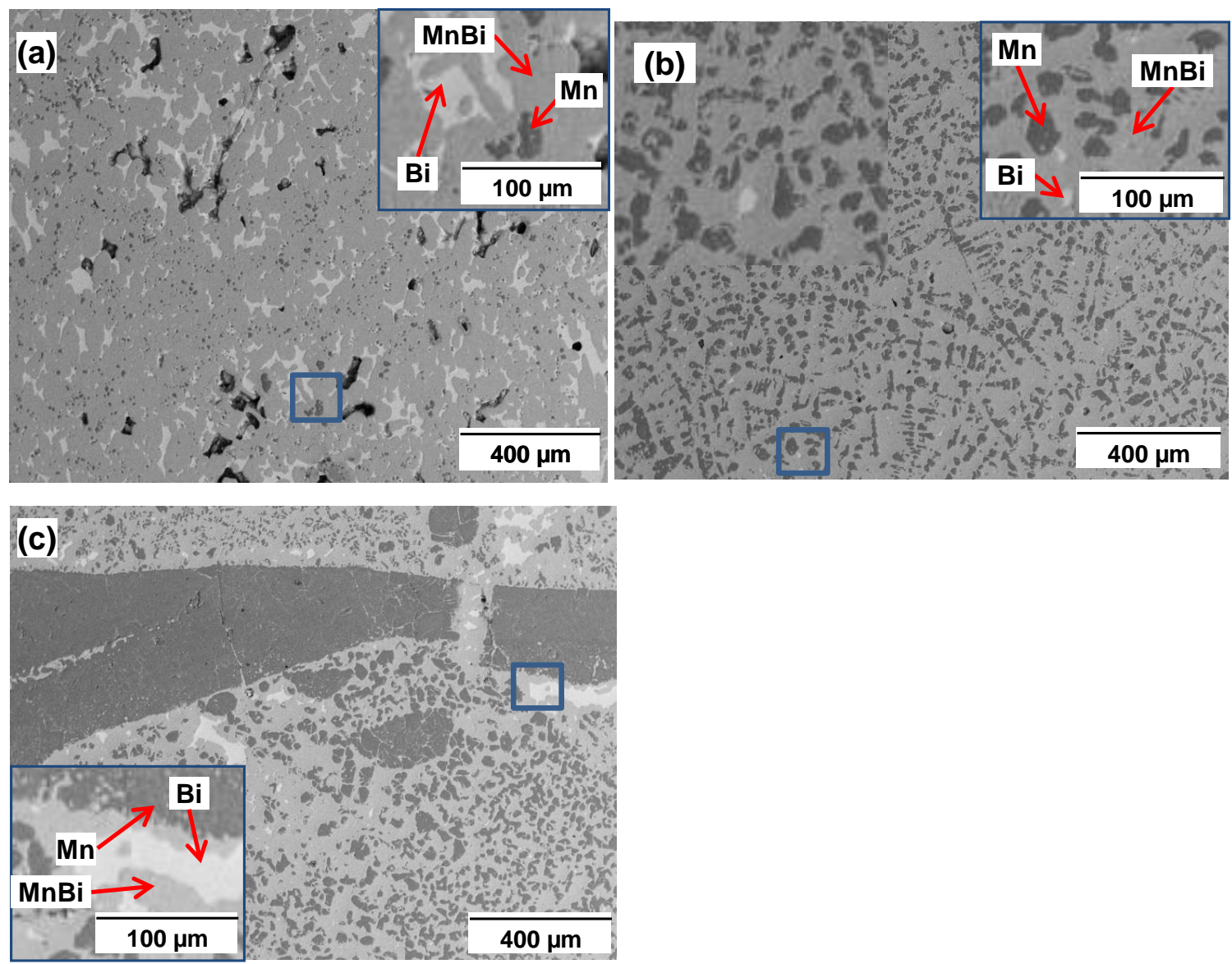

Figure 3. The microstructure of two-step heat-treated samples.

(a) 45 at. $\% \mathrm{Mn}$, (b) 55 at. $\% \mathrm{Mn}$, and (c) 75 at.\% Mn 


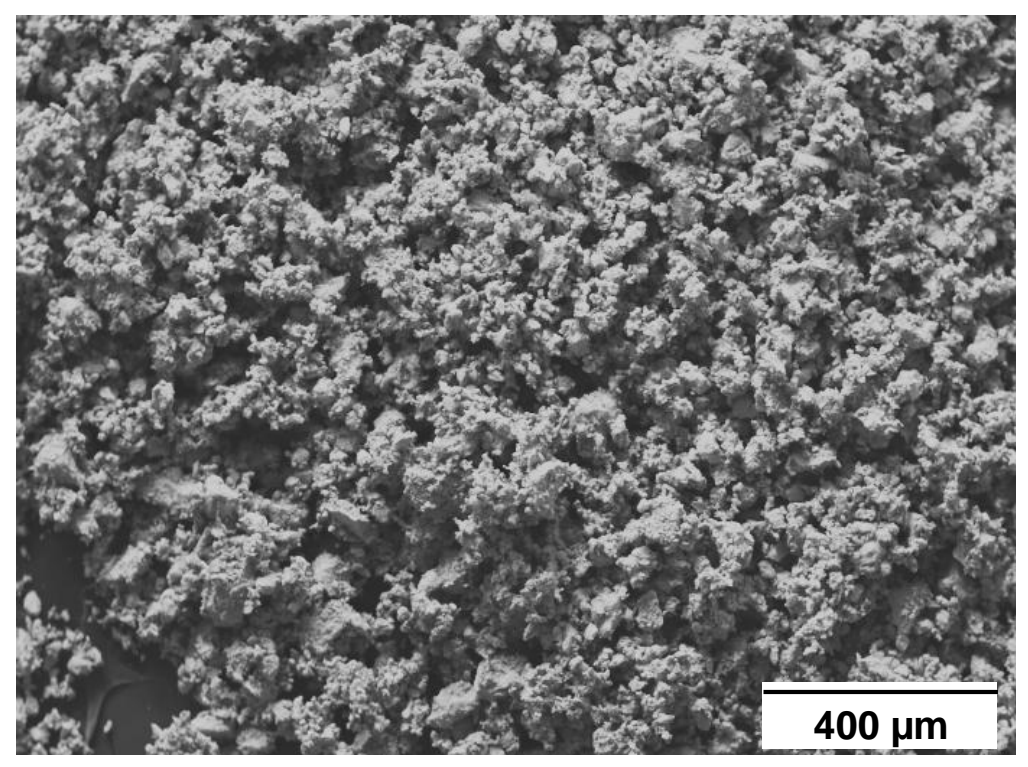

(a)

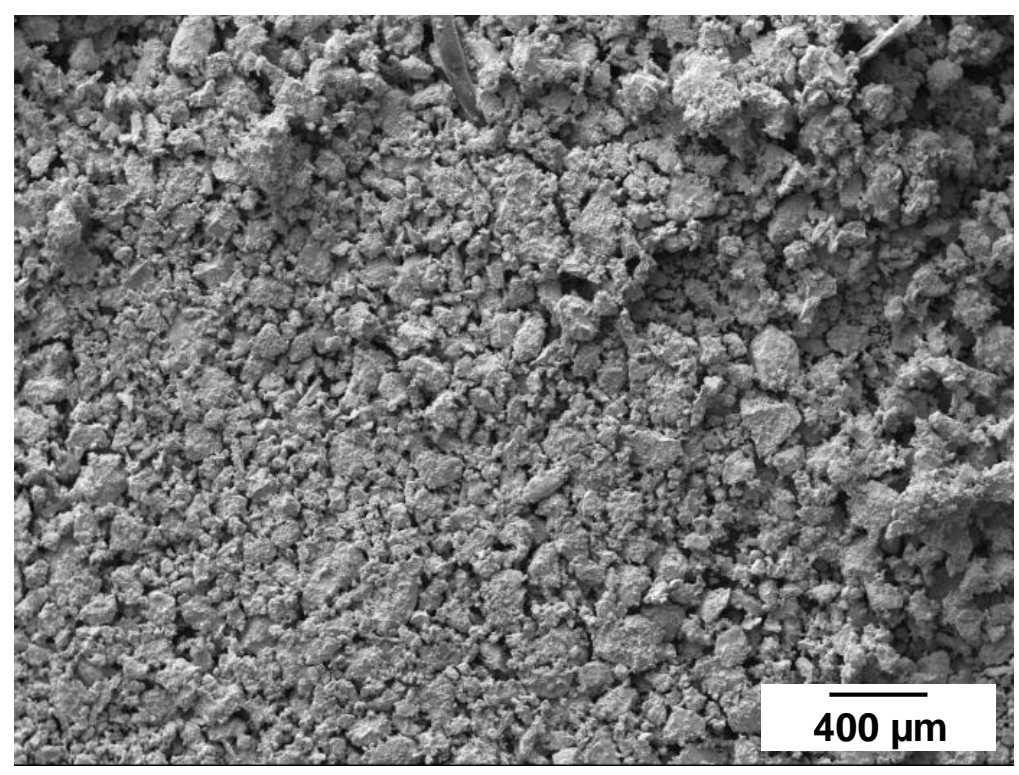

(b)

Figure 4. Powder surface morphology (SEI) before and after vacuum heat treatment (55 at.\% $\mathrm{Mn}$ ); (a) as ground and sieved, (b) after vacuum heat treatment. 


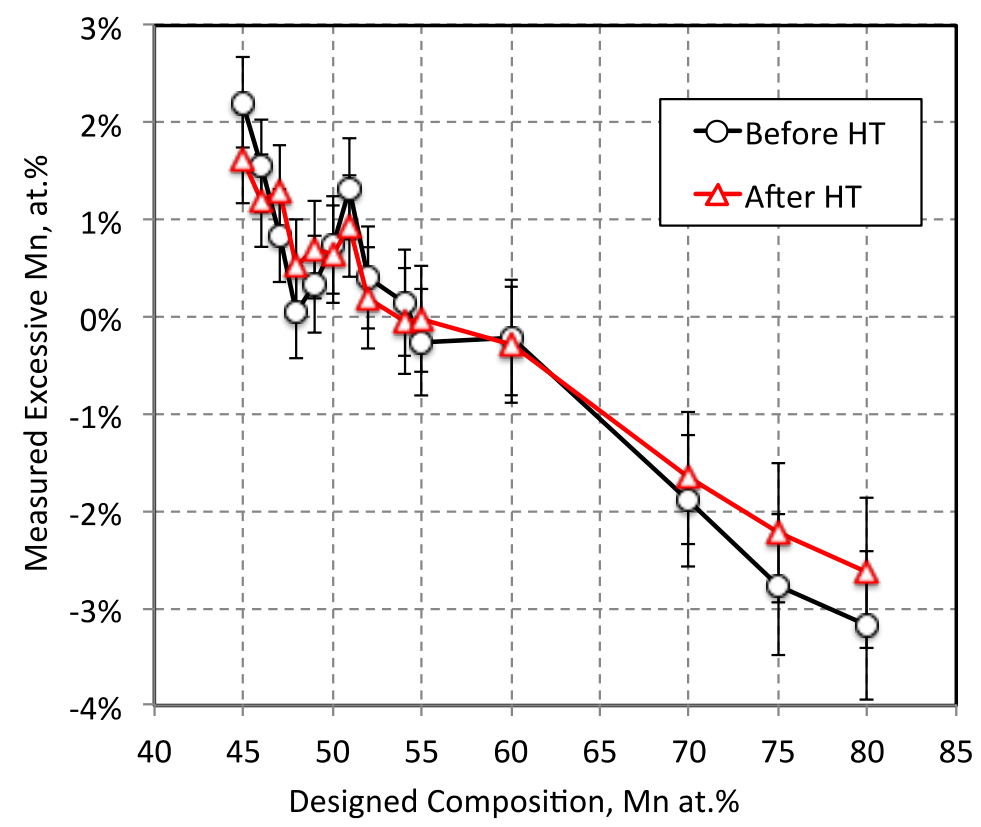

Figure 5. The chemical composition of samples before and after vacuum heat treatment. (Note: "Before HT" is as-ground and sieved sample and $37-44 \mu \mathrm{m}$ size) 


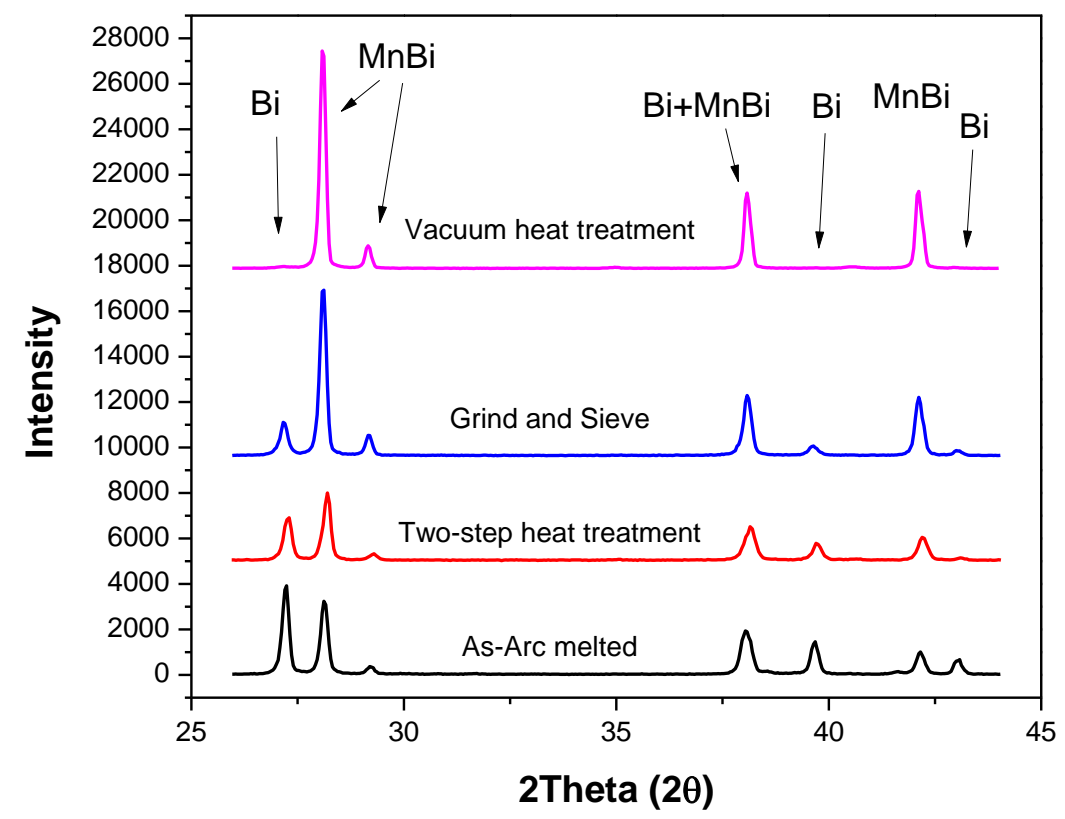

Figure 6. The normalized XRD patterns after each step (55 \pm 1 at. $\% \mathrm{Mn})$. 


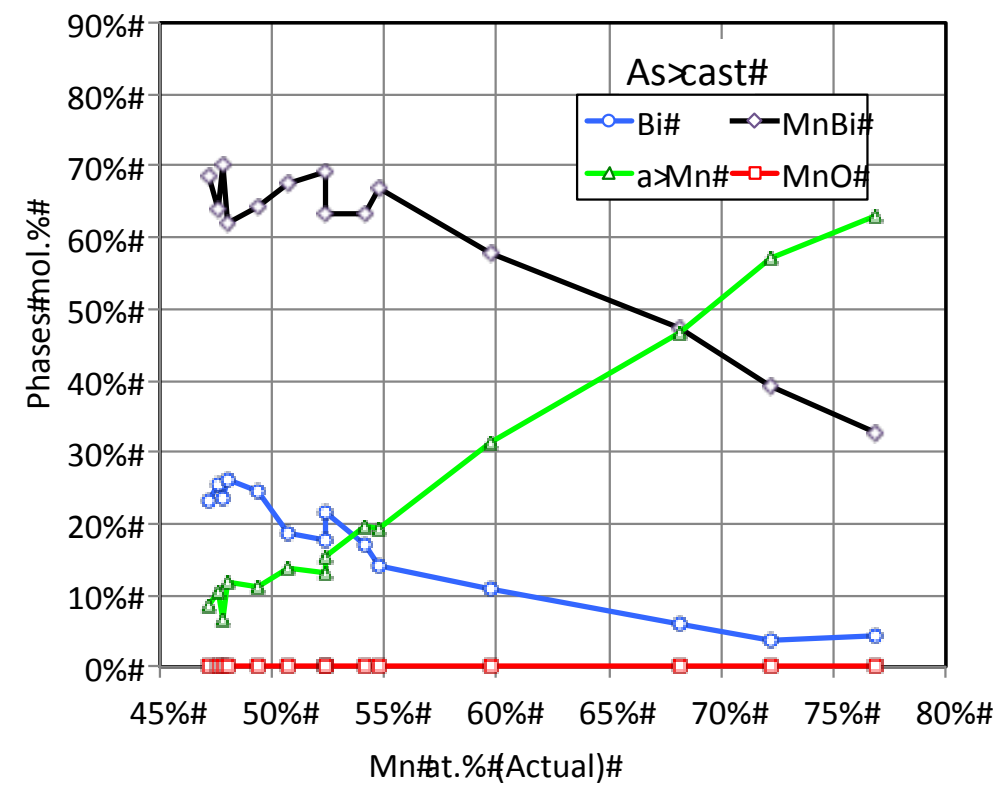

(a)

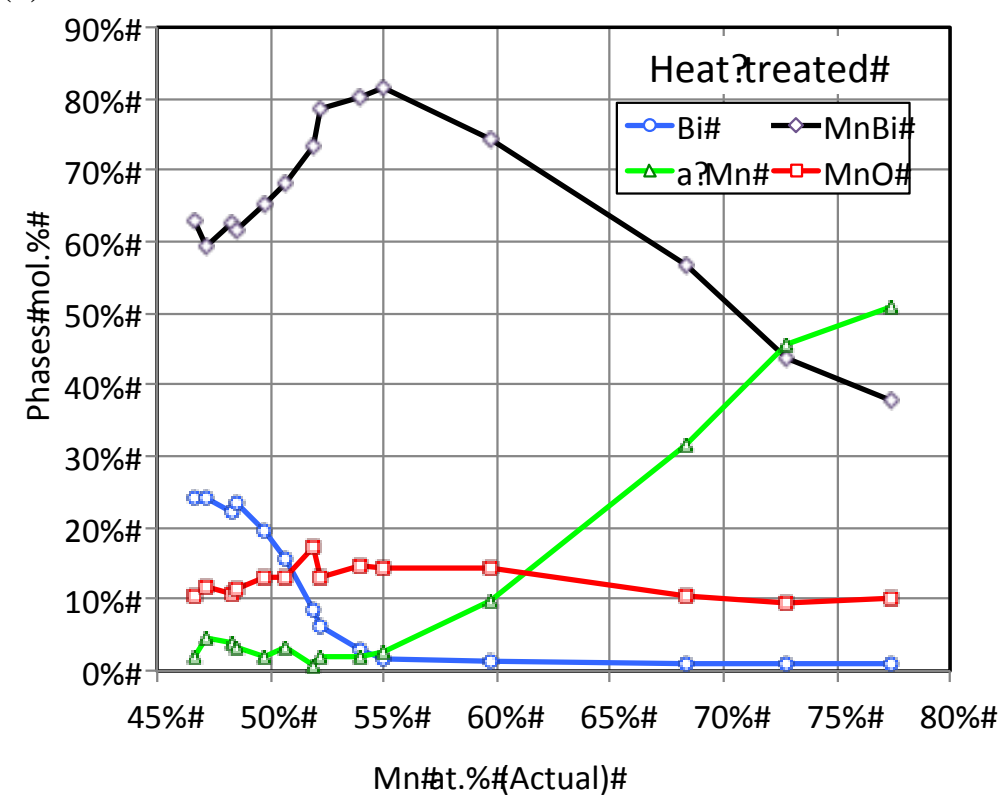

(b)

Figure 7. Phase fractions analysis by XRD (a) before and (b) after vacuum heat treatment.. 


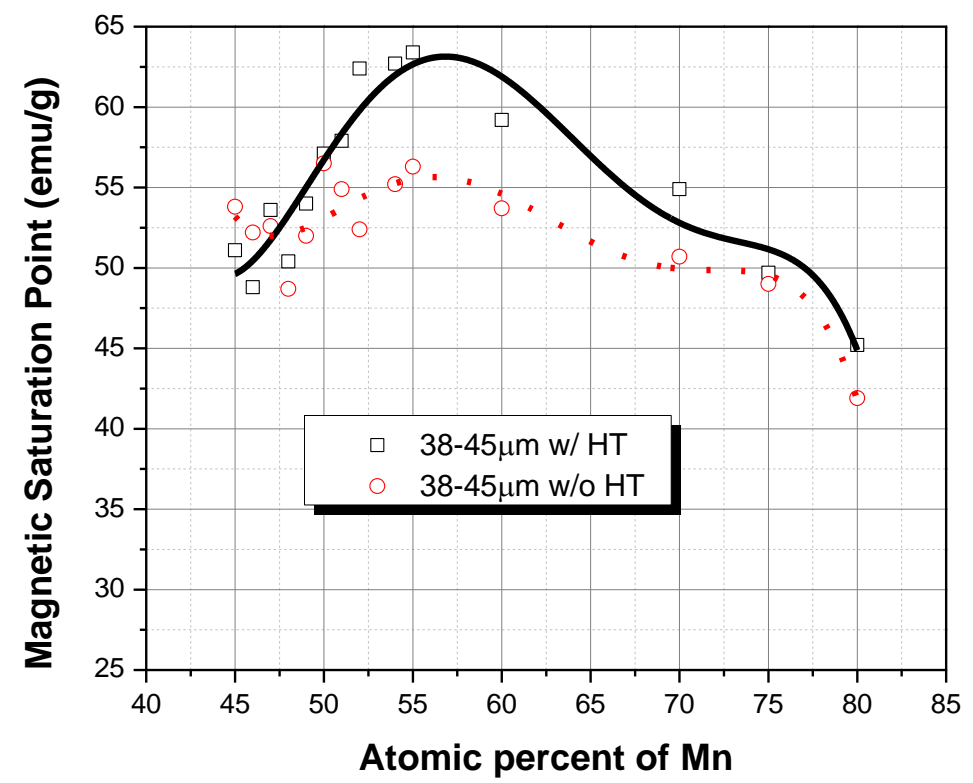

Figure 8. The composition effect on saturation magnetization of the MnBi powders. The magnetizations were measured with $2.3 \mathrm{~T}$ field at $300 \mathrm{~K}$. 


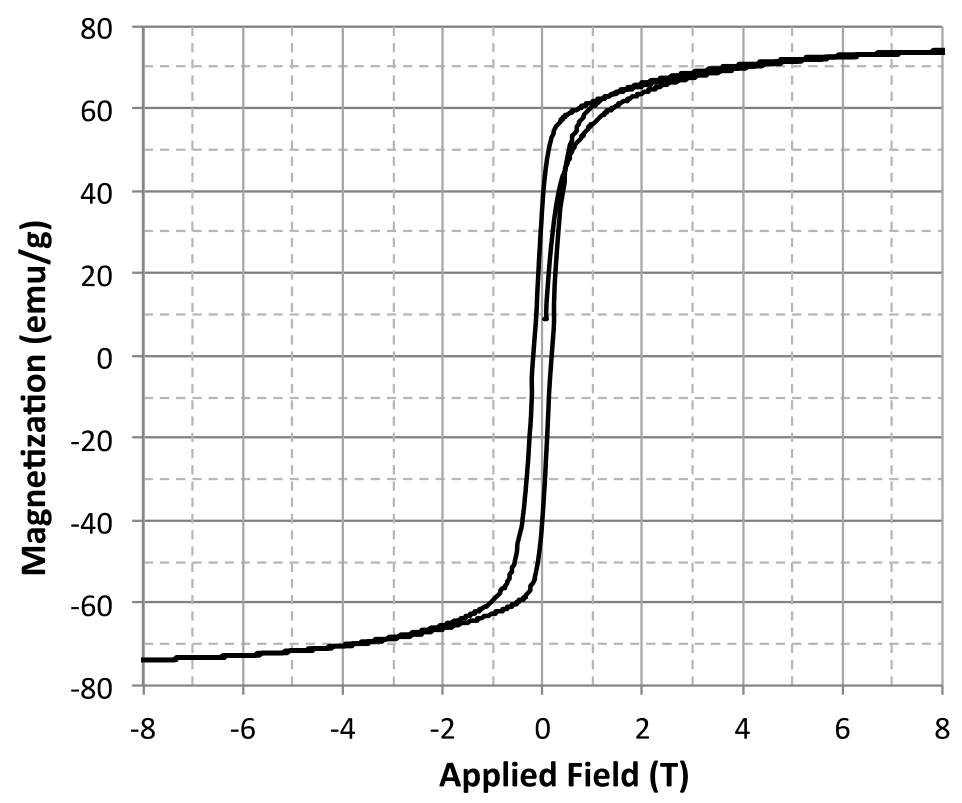

Figure 9. $\mathrm{MH}$ loop of the MnBi powders with 55 at.\% Mn composition. The measurement was carried out at $300 \mathrm{~K}$.

[1] Okamoto H. Bi-Mn phase diagram. Alloy Phase Diagrams. ASM International, 1990. 
Table 1. The chemical composition of samples before and after vacuum heat treatment (by XRD analysis).

\begin{tabular}{l|lllll|lllll}
\multicolumn{9}{|c}{ Before vacuum heat treatment } & \multicolumn{1}{c}{ After vacuum heat treatment } \\
\hline \hline $\begin{array}{l}\text { Designed } \\
\text { at.\% Mn }\end{array}$ & $\begin{array}{l}\text { Actual } \\
\text { at.\% Mn }\end{array}$ & $\begin{array}{l}\text { MnBi } \\
\text { wt.\% }\end{array}$ & $\begin{array}{l}\text { Bi } \\
\text { wt.\% }\end{array}$ & $\begin{array}{l}\alpha-M n \\
\text { wt.\% }\end{array}$ & $\begin{array}{l}\text { MnO } \\
\text { wt.\% }\end{array}$ & $\begin{array}{l}\text { Actual } \\
\text { at.\% Mn }\end{array}$ & $\begin{array}{l}\text { MnBi } \\
\text { wt.\% }\end{array}$ & $\begin{array}{l}\text { Bi } \\
\text { wt.\% }\end{array}$ & $\begin{array}{l}\alpha-M n \\
\text { wt.\% }\end{array}$ & $\begin{array}{l}\text { MnO } \\
\text { wt.\% }\end{array}$ \\
\hline \hline 45 & 47 & 77 & 21 & 2 & 0 & 47 & 74 & 23 & 1 & 3 \\
46 & 48 & 74 & 23 & 3 & 0 & 47 & 72 & 23 & 1 & 4 \\
47 & 48 & 78 & 21 & 2 & 0 & 48 & 75 & 21 & 1 & 4 \\
48 & 48 & 73 & 24 & 3 & 0 & 49 & 73 & 22 & 1 & 4 \\
49 & 49 & 75 & 23 & 3 & 0 & 50 & 77 & 18 & 1 & 4 \\
50 & 51 & 79 & 17 & 3 & 0 & 51 & 81 & 15 & 1 & 4 \\
51 & 52 & 81 & 16 & 3 & 0 & 52 & 86 & 8 & 0 & 6 \\
52 & 52 & 76 & 20 & 4 & 0 & 52 & 90 & 6 & 1 & 4 \\
54 & 54 & 78 & 17 & 5 & 0 & 54 & 92 & 3 & 1 & 5 \\
55 & 55 & 82 & 14 & 5 & 0 & 55 & 93 & 2 & 1 & 4 \\
60 & 60 & 79 & 12 & 9 & 0 & 60 & 91 & 1 & 3 & 5 \\
70 & 68 & 80 & 8 & 16 & 0 & 68 & 85 & 1 & 10 & 4 \\
75 & 72 & 73 & 5 & 22 & 0 & 73 & 77 & 1 & 17 & 5 \\
80 & 77 & 67 & 7 & 27 & 0 & 77 & 73 & 2 & 20 & 5 \\
\hline
\end{tabular}

